\title{
Around some extensions of Casas-Alvero conjecture for non-polynomial functions
}

\author{
A. Cima, A. Gasull, F. Mañosas \\ Departament de Matemàtiques, Universitat Autònoma de Barcelona \\ Barcelona, Spain \\ cima@mat.uab.cat, gasull@mat.uab.cat, manyosas@mat.uab.cat
}

\author{
Received April 21, 2020 \\ Accepted May 2, 2020 \\ Abstract: We show that two natural extensions of the real Casas-Alvero conjecture in the non- \\ polynomial setting do not hold. \\ Key words: polynomial, Casas-Alvero conjecture, zeroes of functions. \\ AMS Subject Class. (2010): Primary: 30C15. Secondary: 12D10, 13P15, $26 \mathrm{C} 10$.
}

Presented by Manuel Maestre

\section{Introduction}

The Casas-Alvero conjecture affirms that if a complex polynomial $P$ of degree $n>1$ shares roots with all its derivatives, $P^{(k)}, k=1,2, \ldots, n-1$, then there exist two complex numbers, $a$ and $b \neq 0$, such that $P(z)=b(z-$ $a)^{n}$. Notice that, in principle, the common root between $P$ and each $P^{(k)}$ might depend on $k$. Casas-Alvero arrived to this problem at the turn of this century, when he was working in his paper [1] trying to obtain an irreducibility criterion for two variable power series with complex coefficients. See [2] for an explanation of the problem in his own words.

Although several authors have got partial answers, to the best of our knowledge the conjecture remains open. For $n \leq 4$ the conjecture is a simple consequence of the wonderful Gauss-Lucas Theorem ([6]). In 2006 it was proved in [5], by using Maple, that it is true for $n \leq 8$. Afterwards in [6, 7] it was proved that it holds when $n$ is $p^{m}, 2 p^{m}, 3 p^{m}$ or $4 p^{m}$, for some prime number $p$ and $m \in \mathbb{N}$. The first cases left open are those where $n=24,28$ or 30 . See again [6] for a very interesting survey or [3, 8] for some recent contributions on this question.

Adding the hypotheses that $P$ is a real polynomial and all its $n$ roots, taking into account their multiplicities, are real, the conjecture has a real 
counterpart, that also remains open. It says that $P(x)=b(x-a)^{n}$ for some real numbers $a$ and $b \neq 0$. For this real case, the conjecture can be proved easily for $n \leq 4$, simply by using Rolle's Theorem. This tool does not suffice for $n \geq 5$, see for instance [4] for more details, or next section.

Also in the real case, in [6] it is proved that if the condition for one of the derivatives of $P$ is removed, then there exist polynomials satisfying the remaining $n-2$ conditions, different from $b(x-a)^{n}$. The construction of some of these polynomials presented in that paper is very nice and is a consequence of the Brouwer's fixed point Theorem in a suitable context.

Finally, it is known that if the conjecture holds in $\mathbb{C}$, then it is true over all fields of characteristic 0 . On the other hand, it is not true over all fields of characteristic $p$, see again [7]. For instance, consider $P(x)=x^{2}\left(x^{2}+1\right)$ in characteristic 5 with roots $0,0,2$ and 3 . Then $P^{\prime}(x)=2 x\left(2 x^{2}+1\right), P^{\prime \prime}(x)=$ $12 x^{2}+2=2\left(x^{2}+1\right)$ and $P^{\prime \prime \prime}(x)=4 x$ and all them share roots with $P$.

The aim of this note is to present two natural extensions of the real CasasAlvero conjecture to smooth functions and show that none of them holds.

Question 1. Fix $1<n \in \mathbb{N}$. Let $F$ be a class $\mathcal{C}^{n}$ real function such that $F^{(n)}(x) \neq 0$ for all $x \in \mathbb{R}$, and has $n$ real zeroes, taking into account their multiplicities. Assume that $F$ shares zeroes with all its derivatives, $F^{(k)}$, $k=1,2, \ldots, n-1$. Is it true that $F(x)=b(f(x))^{n}$ for some $0 \neq b \in \mathbb{R}$ and some $f$, a class $\mathcal{C}^{n}$ real function, that has exactly one simple zero?

Notice that one of the hypotheses of the real Casas-Alvero conjecture can be reformulated as follows: The polynomial $F$ shares roots with all its derivatives but one, precisely the one corresponding to its degree. Trivially, this is so, because all the derivatives of order higher than $n$ are identically zero. The second question that we consider is:

Question 2. Fix $1<n \in \mathbb{N}$. Let $F$ be a real analytic function that shares zeroes with all its derivatives but one, say $F^{(n)}$. Is it true that $F(x)=b(f(x))^{n}$ for some $0 \neq b \in \mathbb{R}$ and some real analytic function $f$, that has exactly one simple zero?

Theorem A. (i) The answer to the Question 1 is "yes" for $n \leq 4$ and "no" for $n=5$.

(ii) The answer to the Question 2 is already "no" for $n=2$.

Our result reinforces the intuitive idea that Casas-Alvero conjecture is mainly a question related with the rigid structure of the polynomials. 


\section{Proof of Theorem $\mathrm{A}$}

(i) The answer to Question 1 is "yes" for $n=2,3,4$ because the proof of the real Casas-Alvero conjecture for the same values of $n$, based on the Rolle's Theorem and given in [4], does not uses at all that $P$ is a polynomial. Let us adapt it to our setting. Since $F^{(n)}$ does not vanish we know that $F$ has exactly $n$ real zeroes, taking into account their multiplicites. Moreover we know that $F$ has to have at least a double zero, that without loss of generality can be taken as 0 . Next we can do a case by case study to discard all situations except that $F$ has only a zero and it is of multiplicity $n$. For the sake of brevity, we give all the details only in the most difficult case, $n=4$.

Assume, to arrive to a contradiction, that $n=4, F$ is under the hypotheses of Question 1 and $x=0$ is not a zero of multiplicity four. Notice that by Rolle's theorem, for $k=1,2,3$, each $F^{(k)}$ has exactly $4-k$ zeroes, taking into account their multiplicities. Moreover, the only zero of $F^{\prime \prime \prime}$ must be one of the zeroes of $F$.

If $F^{\prime \prime}(0)=0$ and $F^{\prime \prime \prime}(0) \neq 0$ then $F$ has only another zero at $x=a$ and, without loss of generality, we can assume that $a>0$. Applying three times Rolle's theorem we get that $F^{\prime \prime \prime}(b)=0$ for some $b \in(0, a)$ which is a contradiction with the hypotheses. If $F^{\prime \prime}(0) \neq 0$ then $F$ has two more zeroes counting multiplicities. There are three possibilities. The first one is that there is $a>0$ such that $F(a)=F^{\prime}(a)=0$. In this case, applying two times Rolle's theorem we obtain that there exist $b, c \in(0, a)$ with $F^{\prime \prime}(b)=F^{\prime \prime}(c)=0$ and they are the only zeroes of $F^{\prime \prime}$. This fact gives again a contradiction because none of them is a zero of $F$. The second one is that there exist $a_{1}, a_{2} \in \mathbb{R}$ with $0 \in\left(a_{1}, a_{2}\right)$ such that $F\left(a_{1}\right)=F\left(a_{2}\right)=0$. Also in this case, by applying two times Rolle's theorem we obtain that there exist $b, c \in\left(a_{1}, a_{2}\right)$ such that $0 \in(b, c)$ and $F^{\prime \prime}(b)=F^{\prime \prime}(c)=0$ giving us the desired contradiction. Lastly, assume that the other two zeroes of $F$ are $a_{1}$ and $a_{2}$, with $0<a_{1}<a_{2}$. By Rolle's Theorem the zeroes of $F^{\prime}$ are $0, b_{1}$ and $b_{2}$ and satisfy $0<b_{1}<a_{1}<$ $b_{2}<a_{2}$. Then, since $F^{\prime \prime}$ has to have two zeroes, say $c_{1}, c_{2}$, and they satisfy $0<c_{1}<b_{1}<c_{2}<b_{2}$, the hypotheses force that $c_{2}=a_{1}$. Hence the zero of $F^{\prime \prime \prime}$ has to be between $c_{1}$ and $c_{2}=a_{1}$, that is in particular in $\left(0, a_{1}\right)$, interval that contains no zero of $F$, arriving once more to the desired contradiction.

In short, we have proved for $n \leq 4$, that $F(x)=x^{n} G(x)$, for some class $\mathcal{C}^{n}$ function $G$, that does not vanish. Hence

$$
F(x)=\operatorname{sign}(G(0))\left(x \sqrt[n]{\frac{G(x)}{\operatorname{sign}(G(0))}}\right)^{n}=b(f(x))^{n},
$$


where $f$ has only one zero, $x=0$, that is simple, as we wanted to prove.

To find a map $F$ for which the answer to Question 1 is "no" we consider $n=5$ and a configuration of zeroes of $F$ and its derivatives proposed in [4] as the simplest one, compatible with the hypotheses of the Casas-Alvero conjecture and Rolle's Theorem. Specifically, we will search for a function $F$, of class at least $\mathcal{C}^{5}$, with the five zeroes $0,0,1, c, d$, to be fixed, satisfying $0<1<c<d$, and moreover

$$
F^{\prime}(0)=0, \quad F^{\prime \prime}(1)=0, \quad F^{\prime \prime \prime}(c)=0, \quad F^{(4)}(1)=0,
$$

and such that $F^{(5)}$ does not vanish. Notice that $F^{\prime}(0)=0$ is not a new restriction.

We start assuming that $F^{(5)}(x)=r-\sin (x)$, for some $r>1$ to be determined. By imposing that conditions (2.1) hold, together with $F(0)=0$, we get that

$$
F(x)=\int_{0}^{x} \int_{0}^{u} \int_{1}^{w} \int_{c}^{z} \int_{1}^{y}(r-\sin (t)) \mathrm{d} t \mathrm{~d} y \mathrm{~d} z \mathrm{~d} w \mathrm{~d} u
$$

Some straightforward computations give that

$$
\begin{aligned}
F(x)= & \frac{r}{120} x^{5}-\frac{r+\cos (1)}{12} x^{4}+\frac{2 r c-2 \sin (c)+2 \cos (1) c-r c^{2}}{12} x^{3} \\
& +\frac{6 \sin (c)+2 r+9 \cos (1)-6 r c+3 r c^{2}-6 \cos (1) c}{12} x^{2}-1+\cos (x) .
\end{aligned}
$$

Imposing now that $F(1)=0$ we obtain that

$$
r=\frac{5(8 \cos (1) c-41 \cos (1)-8 \sin (c)+24)}{4\left(5 c^{2}-10 c+4\right)}=R(c) .
$$

Next we have to impose that $F(c)=0$. By replacing the above expression of $r$ in $F$ we obtain that

$$
F(c)=\frac{G(c)}{96\left(5 c^{2}-10 c+4\right)}
$$

where

$$
\begin{aligned}
G(c)= & -c^{2}\left(12 c^{4}-369 c^{3}+1437 c^{2}-1708 c+532\right) \cos (1) \\
& -8 c^{2}(c-1)(c-2)^{2} \sin (c)+\left(480 c^{2}-960 c+384\right) \cos (c) \\
& -24(c-1)\left(9 c^{4}-36 c^{3}+24 c^{2}+24 c-16\right) .
\end{aligned}
$$


A carefully study shows that $G$ has exactly one real zero $c_{1} \in(17 / 10,19 / 10)$ $=I$, with $c_{1} \approx 1.79343096$. To prove its existence it suffices to show that

$$
\begin{aligned}
G\left(\frac{17}{10}\right)= & -\frac{99211099}{500000} \cos (1)-\frac{18207}{12500} \sin \left(\frac{17}{10}\right) \\
& +\frac{696}{5} \cos \left(\frac{17}{10}\right)+\frac{1583211}{12500}>0, \\
G\left(\frac{19}{10}\right)= & -\frac{180110481}{500000} \cos (1)-\frac{3249}{12500} \sin \left(\frac{19}{10}\right) \\
& +\frac{1464}{5} \cos \left(\frac{19}{10}\right)+\frac{3616677}{12500}<0 .
\end{aligned}
$$

By using Taylor's formula we know that for any $c>0, S^{-}(c)<\sin (c)<S^{+}(c)$ and $C^{-}(c)<\cos (c)<C^{+}(c)$ where

$$
S^{ \pm}(c)=c-\frac{c^{3}}{3 !}+\frac{c^{5}}{5 !}-\frac{c^{7}}{7 !}+\frac{c^{9}}{9 !} \pm \frac{c^{11}}{11 !}
$$

and

$$
C^{ \pm}(c)=1-\frac{c^{2}}{2 !}+\frac{c^{4}}{4 !}-\frac{c^{6}}{6 !}+\frac{c^{8}}{8 !} \pm \frac{c^{10}}{10 !}
$$

Hence we can replace the values of the trigonometric functions in $G$ by rational numbers to have upper or lower bounds of this function evaluated at $1,17 / 10$ or $19 / 10$. For instance,

$$
0.5403023 \approx \frac{1960649}{3628800}=C^{-}(1)<\cos (1)<C^{+}(1)=\frac{280093}{518400} \approx 0.5403028 .
$$

We obtain that

$$
\begin{aligned}
G\left(\frac{17}{10}\right)> & -\frac{99211099}{500000} C^{+}(1)-\frac{18207}{12500} S^{+}\left(\frac{17}{10}\right)+\frac{696}{5} C^{-}\left(\frac{17}{10}\right) \\
& +\frac{1583211}{12500}=\frac{3444600099561969856969}{49896000000000000000000}>0
\end{aligned}
$$

and

$$
\begin{aligned}
G\left(\frac{19}{10}\right)< & -\frac{180110481}{500000} C^{-}(1)-\frac{3249}{12500} S^{-}\left(\frac{19}{10}\right)+\frac{1464}{5} C^{+}\left(\frac{19}{10}\right) \\
& +\frac{3616677}{12500}=-\frac{1689627895469649855823}{16632000000000000000000}<0 .
\end{aligned}
$$


To show the uniqueness of the zero in $I$, we will prove that $G$ is strictly decreasing in this interval. It holds that

$$
G^{\prime}(c)=T(c) \cos (1)+U(c) \sin (c)+V(c \cos (c)+W(c),
$$

with

$$
\begin{aligned}
T(c) & =-c\left(72 c^{4}-1845 c^{3}+5748 c^{2}-5124 c+1064\right), \\
U(c) & =-8\left(5 c^{2}-10 c+4\right)\left(c^{2}-2 c+12\right), \\
V(c) & =-8(c-1)\left(c^{4}-4 c^{3}+4 c^{2}-120\right) \\
W(c) & =-120\left(9 c^{4}-36 c^{3}+36 c^{2}-8\right) .
\end{aligned}
$$

By computing the Sturm sequences of $T, U$ and $V$ we can prove that $T(c)<0$, $U(c)<0$ and $V(c)>0$ for all $c \in I$. Hence, for $c \in I$,

$$
G^{\prime}(c)<T(c) C^{-}(c)+U(c) S^{-}(c)+V(c) C^{+}(c)+W(c)=Q(c),
$$

where

$$
\begin{aligned}
Q(c)= & \frac{72469}{64800} c-\frac{669211}{43200} c^{2}+\frac{18852329}{302400} c^{3}-\frac{8854991}{80640} c^{4} \\
& +\frac{4732471}{50400} c^{5}-\frac{532}{15} c^{6}+\frac{8}{7} c^{7}+\frac{191}{70} c^{8} \\
& -\frac{797}{1890} c^{9}-\frac{34}{405} c^{10}+\frac{1651}{103950} c^{11}+\frac{3533}{2494800} c^{12} \\
& -\frac{193}{623700} c^{13}+\frac{1}{142560} c^{14}-\frac{1}{831600} c^{15} .
\end{aligned}
$$

The Sturm sequence of $Q$ shows that it has no zeroes in $I$. Moreover, it is negative in this interval, and as a consequence, $G^{\prime}$ is also negative, as we wanted to prove.

We fix $c=c_{1}$. Then, $r=R\left(c_{1}\right)$ and $F$ is also totally fixed. Moreover, by using the same techniques we get that $r=R\left(c_{1}\right)>R(19 / 10)>1$ and as a consequence $F^{(5)}$ does not vanish. In fact, $r=R\left(c_{1}\right) \approx 1.04591089$. Finally, $F$ has one more real zero $d \in(33 / 10,34 / 10)$. In fact, $d \approx 3.32178369$. This $F$ gives our desired example, see Figure 1.

(ii) Consider $F(x)=4 x^{2}+\pi^{2}(\cos (x)-1)$ that has a double zero at 0 and also vanishes at $\pm \pi / 2$. Moreover, $F^{\prime}(x)=8 x-\pi^{2} \sin (x)$ vanishes at $x=0$, $F^{\prime \prime}(x)=8-\pi^{2} \cos (x)$ has no common zeroes with $F$ and, for any $k>1$, 
$\left|F^{(2 k)}(x)\right|=\pi^{2}|\cos (x)|$ vanishes at $x=\pi / 2$ and $\left|F^{(2 k-1)}(x)\right|=\pi^{2}|\sin (x)|$ vanishes at $x=0$.

A similar example for $n=3$ is $F(x)=4 x^{3}-6 \pi x^{2}+\pi^{3}(1-\cos (x))$, that vanishes at $0, \pi$ (double zeroes) and $\pi / 2$.

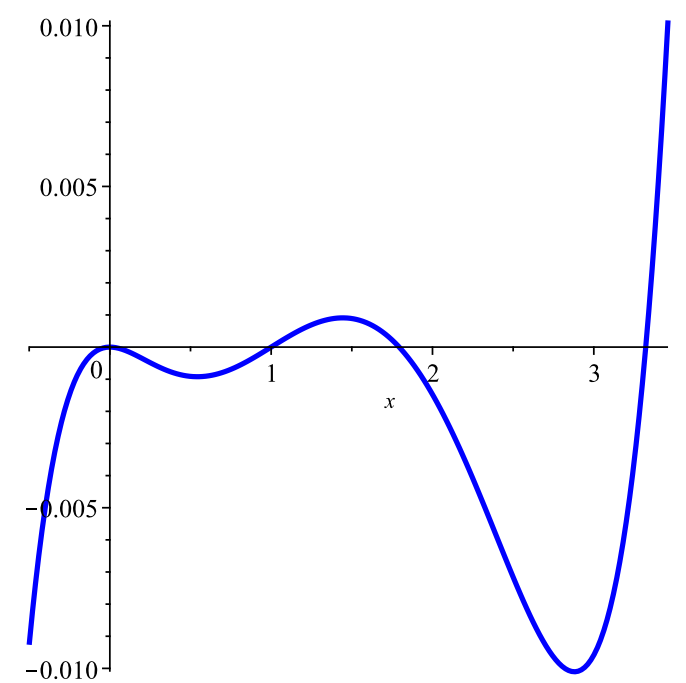

Figure 1: Plot of a map $F$ for which the answer to Question 1 for $n=5$ is "no".

\section{ACKNOWLEDGEMENTS}

The authors are supported by Ministerio de Ciencia, Innovación y Universidades of the Spanish Government through grants MTM201677278-P (MINECO/AEI/FEDER, UE, first and second authors) and MTM2017-86795-C3-1-P (third author). The three authors are also supported by the grant 2017-SGR-1617 from AGAUR, Generalitat de Catalunya.

\section{REFERENCES}

[1] E. Casas-Alvero, Higher order polar germs, J. Algebra 240 (2001), $326-337$.

[2] Interview to E. Casas-Alvero in Spanish. https://www.gaussianos.com/la-conjetura-de-casas-alvero-contada -por-eduardo-casas-alvero/. 
[3] W. Castryck, R. Laterveer, M. Ounä̈es, Constraints on counterexamples to the Casas-Alvero conjecture and a verification in degree 12, Math. Comp. 83 (2014), 3017-3037.

[4] M. Chellali, On the number of real polynomials of the Casas-Alvero type, J. of Taibah Univ. for Science 9 (2015), 351-356.

[5] G.M. DíAz-Toca, L. GonzÁlez-VeGA, On analyzing a conjecture about univariate polynomials and their roots by using Maple in "A Maple conference 2006" (Proc. of the conference; Waterloo, Ontario, Canada, July 23-26, 2006; Waterloo: Maplesoft), 2006, 81-98.

[6] J. Draisma, J.P. De Jong, On the Casas-Alvero conjecture, Eur. Math. Soc. Newsl. 80 (2011), 29-33.

[7] H.-C. Graf von Bothmer, O. Labs, J. Schicho, C. van de Woestijne, The Casas-Alvero conjecture for infinitely many degrees, $J$. Algebra 316 (2007), 224-230.

[8] S. Yakubovich, Polynomial problems of the Casas-Alvero type, J. Class. Anal. 4 (2014), 97-120. 